\title{
Universal scaling of the conductivity relaxation in crystalline ionic conductors
}

\author{
C. León, M. L. Lucía, J. Santamaría, and F. Sánchez-Quesada \\ Departamento de Física Aplicada III, Facultad de Ciencias Físicas, Universidad Complutense de Madrid, Avda. Complutense s/n, \\ 28040 Madrid, Spain \\ (Received 31 July 1997)
}

\begin{abstract}
We present complex admittance measurements on single-crystal yttria-stabilized zirconia and polycrystalline $\mathrm{Li}_{0.5} \mathrm{La}_{0.5} \mathrm{TiO}_{3}$ over the frequency range $5 \mathrm{~Hz}$ to $30 \mathrm{MHz}$ and at temperatures ranging between 150 and $650 \mathrm{~K}$. Electric-field relaxation in both fast ionic conductors can be described using Kohlrausch-Williams-Watts decay functions, but departures are observed at high frequencies and low temperatures. Electric modulus data obey the Dixon-Nagel scaling that has been proposed to be universal in describing the relaxation processes in supercooled liquids. Our data provide broader universality to the Dixon-Nagel scaling, and are interpreted in terms of the influence of mobile ions positional disorder on the relaxation dynamics. [S0163-1829(98)06701-0]
\end{abstract}

Much interest has been paid in recent years to the study of the effect of ion-ion or ion-lattice correlations on the dynamic response of ionic conductors. In this field, electrical conductivity relaxation from complex admittance measurements is one of the most frequently used tools to characterize the effect of cooperativity on ion motion. It turns out that many-body interactions give rise to non-Debye response functions, ${ }^{1-3}$ and these functions are usually well reproduced using stretched exponential relaxation functions of the Kohlrausch-Williams-Watts (KWW) (Ref. 4) kind, i.e., $\phi(t)=e^{-(t / \tau)^{\beta}}$, with the exponent $\beta$ comprised between 0 and 1.

KWW functions have proven to describe the relaxation processes in very different classes of disordered systems ranging from electric-field relaxation in glasses, ${ }^{5}$ or stress relaxation in glasses, ${ }^{6}$ to dielectric relaxation in glassforming polymers ${ }^{7}$ and supercooled liquids, ${ }^{8}$ etc., at least over a few orders of magnitude of frequency around the relaxation peak. However, a description of the relaxation process over wide frequency and temperature ranges is often impossible using a KWW function: significant departures from this behavior have been frequently observed at short times compared with the relaxation time $\tau .{ }^{9}$ In this context an universal scaling of the $\alpha$ relaxation in supercooled liquids has been proposed, ${ }^{8}$ showing that data of different materials over wide frequency and temperature ranges fall on a single scaling curve. The scaling curve clearly shows the departure from the KWW behavior at high frequencies. This scaling has also shown to be valid in other systems like orientationally disordered crystals, ${ }^{10}$ and spin glasses. ${ }^{11}$

In this paper we present data of conductivity relaxation in two crystalline ionic conductors: single-crystal yttriastabilized zirconia and polycrystalline $\mathrm{Li}_{0.5} \mathrm{La}_{0.5} \mathrm{TiO}_{3}$. We show that electric modulus plots may also be normalized using the Dixon-Nagel scaling procedure. ${ }^{8}$ It points to a broader degree of universality of this scaling plot and, besides, may shed some light on the nature of the ionic conduction process in crystalline solids and the role played by the positional disorder of mobile ions in fast ionic conductors. $^{12}$

We have measured complex admittance over the frequency range $5 \mathrm{~Hz}-30 \mathrm{Mhz}$ using standard impedance ana- lyzers (HP 4192A, HP 4284A, and HP 4285A) and at temperatures comprised between 150 and $650 \mathrm{~K}$. Samples were cylindrical cold-pressed pellets in the case of $\mathrm{Li}_{0.5} \mathrm{La}_{0.5} \mathrm{TiO}_{3}$ and $10 \times 5 \times 0.5 \mathrm{~mm},[100]$ oriented, single crystals in the case of yttria-stabilized zirconia (YSZ) $\left(9.5 \% \mathrm{~mol}_{2} \mathrm{O}_{3}\right)$. Contacts were evaporated gold spots. Further experimental details can be found elsewhere. ${ }^{13,14}$

Figure 1 shows the imaginary part of the electric modulus as a function of frequency for both samples. For clarity not all measured temperatures are displayed in the figure. Electric modulus, $M^{*}(\omega)$, is related to the relaxation function through

$$
M^{*}(\omega)=\frac{j \omega \Phi(\omega)}{\epsilon_{\infty}},
$$

where $\Phi(\omega)$ is the Fourier transform of the relaxation function $\phi(t)$, and $\epsilon_{\infty}$ is the high-frequency permittivity. An analysis of modulus vs frequency plots reveals that, near the peak, the relaxation could be described by a KWW relaxation function, being its shape practically temperatureindependent. Beta values of 0.4 were obtained at temperatures at which the peak could be resolved. However, at frequencies well above the peak frequency, clear departures from the KWW behavior (dotted lines in Fig. 1) can be observed, which basically consist of the imaginary part of the modulus becoming almost independent of frequency. Departures from KWW functions can be further discussed in the frame of the conductivity formalism.

It is well known that KWW responses in the time domain can be alternatively described by using power-law dependences on frequency for the complex conductivity $\sigma^{*}(\omega)$, of the form

$$
\sigma^{*}(\omega)=\sigma_{0}\left[1+\left(j \omega / \omega_{c}\right)^{(1-\beta)}+j \omega / \omega_{c}\right],
$$

where $\omega_{c}$ defines the crossover frequency between the constant dc conductivity $\sigma_{0}$ at low frequencies, and the powerlaw dependence at high frequencies. This crossover frequency is related to the relaxation time of the $\mathrm{KWW}$ relaxation function through the expression

$$
\omega_{c}=\frac{\sigma_{0}}{\epsilon_{\infty}}=\frac{\beta}{\Gamma(1 / \beta) \tau},
$$



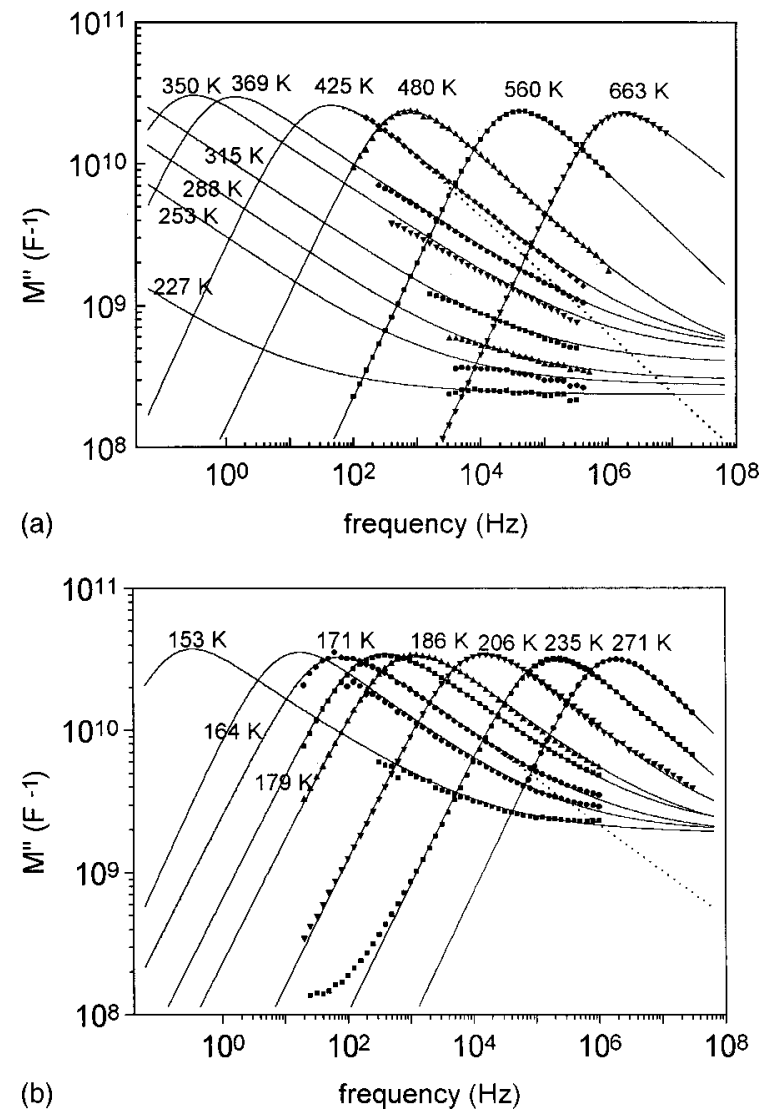

FIG. 1. Frequency dependence of the imaginary part of the electric modulus for (a) YSZ between 227 and $663 \mathrm{~K}$, and for (b) $\mathrm{Li}_{0.5} \mathrm{La}_{0.5} \mathrm{TiO}_{3}$ between 153 and $271 \mathrm{~K}$. In both cases symbols are experimental data and solid lines are fits to expression (4). Dashed lines represent a KWW behavior with $\beta=0.4$; departures from this behavior are observed at high frequencies and low temperatures.

where $\Gamma$ represents the Euler's $\gamma$ function. The equivalence between both pictures in time and frequency domains has been extensively discussed previously in these materials. ${ }^{13,14}$

Figure 2 presents the frequency dependence of the real part of the conductivity, at several temperatures, for the two systems analyzed. It can be observed that, at high frequencies and low temperatures, a new power-dependent contribution appears with an exponent close to one, such that conductivity can then be described as

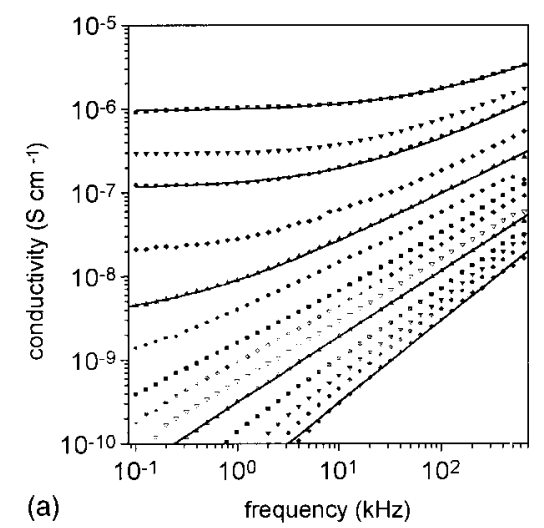

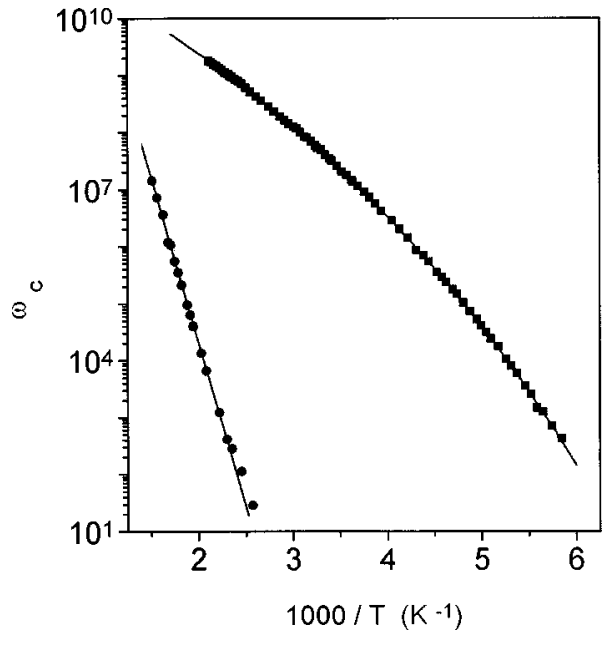

FIG. 3. Temperature dependence of the crossover frequency $\omega_{c}$ for YSZ (O) and $\mathrm{Li}_{0.5} \mathrm{La}_{0.5} \mathrm{TiO}_{3}(\boldsymbol{\square})$. It can be seen the nonArrhenius temperature dependence for $\mathrm{Li}_{0.5} \mathrm{La}_{0.5} \mathrm{TiO}_{3}$. In the case of YSZ, an Arrhenius behavior is obtained in our experimental temperature range.

$$
\sigma^{*}(\omega)=\sigma_{0}\left[1+\left(j \omega / \omega_{c}\right)^{(1-\beta)}+j \omega / \omega_{c}\right]+A \omega .
$$

The temperature dependence of the crossover frequency $\omega_{c}$, which is proportional to de conductivity $\sigma_{0}$, is presented for both systems in Fig. 3 in an Arrhenius plot. In the case of $\mathrm{Li}_{0.5} \mathrm{La}_{0.5} \mathrm{TiO}_{3}$ the behavior is clearly non-Arrhenius; in fact, the line in the figure is a fit to a Vogel-TammanFulcher function, ${ }^{15} \omega_{c}=\omega_{\infty} \exp \left[-A /\left(T-T_{0}\right)\right]$, with $A$ $=1990 \mathrm{~K}$ and $T_{0}=73.3 \mathrm{~K}$. But in the case of YSZ the crossover frequency $\omega_{c}$ remains Arrhenius over the measuring temperature range with an activation energy of $1.16 \mathrm{eV}$, although other authors have previously reported a nonArrhenius behavior from dc conductivity measurements when this range is extended towards high enough temperatures. ${ }^{16}$

Concerning the linear regime at high frequencies, it has been previously observed in different materials and proposed to be a universal feature in ionic conductors. ${ }^{17}$ Its origin has been discussed in terms of localized dipolelike oscillation of ions in asymmetric double-well potentials arising from a disordered distribution of ions. ${ }^{18}$ This concept probably applies

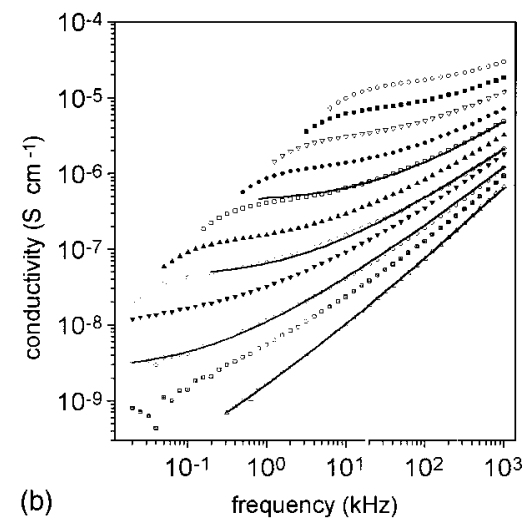

FIG. 2. (a) Frequency dependence of the real part of the electrical conductivity for YSZ between 211 and $560 \mathrm{~K}$; solid lines are fits to expression (4) at $211,332,450,514$, and $560 \mathrm{~K}$. (b) Frequency dependence of the real part of the electrical conductivity for $\mathrm{Li}_{0.5} \mathrm{La}_{0.5} \mathrm{TiO}_{3}$ between 153 and $242 \mathrm{~K}$; solid lines are fits to expression (4) at 153, 171, 186, and $202 \mathrm{~K}$. 
here as a consequence of randomly distributed oxygen vacancies (in YSZ) or lithium ions (in $\mathrm{Li}_{0.5} \mathrm{La}_{0.5} \mathrm{TiO}_{3}$ ). This linear term describes the departures from the KWW relaxation function in the conductivity formalism. Since, as quoted above, fitting conductivity plots to expression (4) allows obtaining all relaxation parameters, modulus plots can be reconstructed using the relation $M^{*}(\omega)=j \omega / \sigma^{*}(\omega)$ even at those temperatures that the peak frequency is out of our measuring frequency window. Solid lines in Fig. 1 are the imaginary part of the electric modulus calculated from complex conductivity fits. It becomes clear from these lines that the departures from KWW relaxation functions observed in modulus plots are due to the onset of the term linear in frequency observed in conductivity plots.

We have shown so far that the relaxation process cannot be described in the whole frequency and temperature range using a single KWW function. In this context, it has been proposed $^{8,10}$ that, instead of searching for the correct relaxation function describing experimental data, an alternative approach is to look at the universality of its general shape for different materials over broad frequency and temperature ranges. Dixon et al. ${ }^{8}$ have shown that a universal scaling curve can be obtained for the dielectric relaxation of different glass formers if one plots $w^{-1} \log _{10}\left(\epsilon^{\prime \prime} f_{p} / \Delta \epsilon f\right)$ vs $w^{-1}\left(1+w^{-1}\right) \log _{10}\left(f / f_{p}\right) . w$ is the width of the susceptibility peak normalized to a Debye relaxation, $\Delta \epsilon$ is the relaxation strength, and $f_{p}$ is the peak frequency for the maximum observed in $\epsilon^{\prime \prime}$. Taking into account that, in the case of the conductivity relaxation, electric field relaxes at constant displacement vector, the quantity describing the relaxation is the electric modulus instead of the complex permittivity. ${ }^{12,19}$ A scaling plot has then been constructed displaying plots $w^{-1} \log _{10}\left(M^{\prime \prime} f_{p} / \Delta M f\right)$ vs $w^{-1}\left(1+w^{-1}\right) \log _{10}\left(f / f_{p}\right)$, where $M^{\prime \prime}$ is the imaginary part of the modulus and $\Delta M$ is the modulus relaxation strength. Results are presented in Fig. 4 showing that data of both materials satisfactorily collapse on the same scaling curve. Moreover, the scaling curve obtained has exactly the same form as that reported for other types of relaxation processes in structural glasses. In the same figure the line is a stretched exponential with $\beta=0.4$, showing the departures from this behavior observed at enough high frequencies, and which we have interpreted as due to the onset of the linear regime shown in conductivity vs frequency plots.

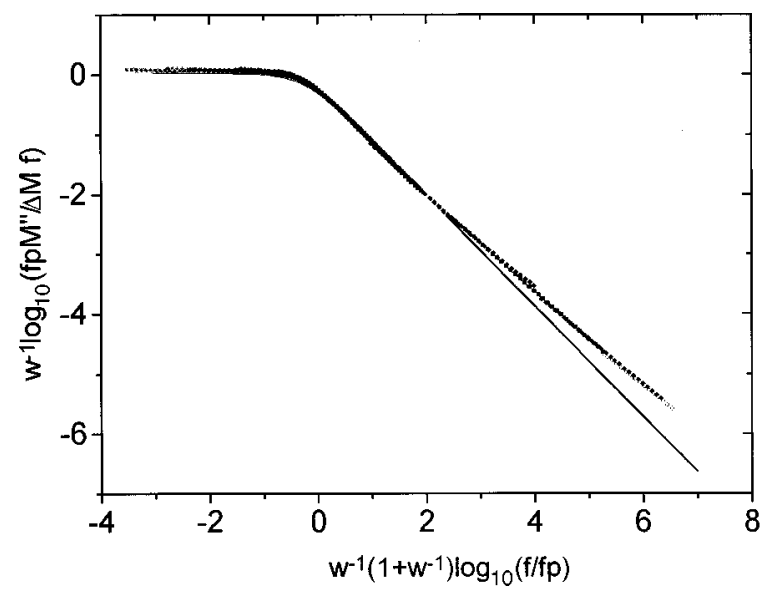

FIG. 4. Dixon-Nagel scaling plot of electrical modulus data describing the electrical conductivity relaxation on YSZ and $\mathrm{Li}_{0.5} \mathrm{La}_{0.5} \mathrm{TiO}_{3}$. The solid line in the figure represents a KWW relaxation with $\beta=0.4$, showing that data depart from this behavior at high frequencies and low temperatures.

Interestingly, our data on conductivity relaxation show many of the characteristic features exhibited by glassy materials in the neighborhood of the glass transition: nonexponential relaxation (KWW), departures from this relaxation function at short times, and non-Arrhenius behavior for the relaxation time. Besides, the fact that electric conductivity relaxation in crystalline ionic conducting solids obeys the same scaling that the dielectric susceptibility in glass forming liquids is remarkable, and shows not only that the DixonNagel scaling has broader universality but also that the nature of the conductivity relaxation in ionic conductors has a complexity beyond the expectations from the crystalline translational order. In fast ionic conductors, glassy or crystalline, the number of available sites exceeds the number of mobile ions and they cannot be unambiguously arranged in an optimized manner, in this sense ionic conductors are said to be characterized by positional disorder. ${ }^{12}$ The dynamics of the ionic diffusion process seem to be governed by cooperative effects among charge carriers and, therefore, the glasslike properties found in the electrical conductivity relaxation of crystalline ionic conductors might be related to positional disorder.
${ }^{1}$ K. L. Ngai, Comments Solid State Phys. 9, 121 (1979); 9, 141 (1980); for a recent review, see K. L. Ngai, in Effects of Disorder on Relaxational Processes, edited by R. Richert and A. Blumen (Springer-Verlag, Berlin, 1994), p. 89.

${ }^{2}$ K. L. Ngai, A. K. Jonscher, and C. T. White, Nature (London) 277, 185 (1979).

${ }^{3}$ K. L. Ngai, Phys. Rev. B 48, 13481 (1993).

${ }^{4}$ R. Kohlrausch, Ann. Phys. (Leipzig) 72, 393 (1847).

${ }^{5}$ C. T. Moynihan, L. P. Boesch, and N. L. Laberge, Phys. Chem. Glasses 14, 122 (1973).

${ }^{6}$ R. Böhmer, H. Senapati, and C. A. Angell, J. Non-Cryst. Solids 131-133, 192 (1991).

${ }^{7}$ J. Colmenero, A. Alegría, J. M. Alberdi, F. Alvarez, and B. Frick, Phys. Rev. B 44, 7321 (1991).
${ }^{8}$ P. K. Dixon, L. Wu, S. R. Nagel, B. D. Williams, and J. P. Carini, Phys. Rev. Lett. 65, 1108 (1990); 66, 959 (1991).

${ }^{9}$ R. V. Chamberlin, R. Böhmer, E. Sánchez, and C. A. Angell, Phys. Rev. B 46, 5787 (1992); R. V. Chamberlin, Europhys. Lett. 33(7), 5454 (1996).

${ }^{10}$ D. L. Leslie-Pelecky and N. O. Birge, Phys. Rev. Lett. 72, 1232 (1994).

${ }^{11}$ D. Bitko, N. Menon, S. R. Nagel, T. F. Rosenbaum, and G. Aeppli, Europhys. Lett. 33, 489 (1996).

${ }^{12}$ K. Funke, Prog. Solid State Chem. 22, 111 (1993).

${ }^{13}$ C. León, M. L. Lucía, J. Santamaría, M. A. París, J. Sanz, and A. Várez, Phys. Rev. B 54, 184 (1996).

${ }^{14}$ C. León, M. L. Lucía, and J. Santamaría, Phys. Rev. B 55, 882 (1997). 
${ }^{15}$ H. Vogel, Phys. Z. 22, 645 (1921); G. S. Fulcher, J. Am. Ceram. Soc. 8, 339 (1925); G. Tamman and W. Hesse, Z. Anorg. Chem. 156, 245 (1926).

${ }^{16}$ J. D. Solier, I. Cachadiña, and A. Dominguez-Rodriguez, Phys. Rev. B 48, 3704 (1993).
${ }^{17}$ W. K. Lee, J. F. Liu, and A. S. Nowick, Phys. Rev. Lett. 67, 1559 (1991).

${ }^{18}$ O. Kanert, J. Steinert, H. Jain, and K. L. Ngai, J. Non-Cryst. Solids 130, 1001 (1991).

${ }^{19}$ P. B. Macedo, C. T. Moynihan, and R. Bose, Phys. Chem. Glasses 13, 171 (1972). 